\title{
Electrocardiography: Flecainide Toxicity
}

Jonathan D. Apfelbaum, MD, FACEP; Jessica Gerczynski; William E. Robertson, DHSc; Stephen Richey, BS

\section{An 86-year-old woman presented to the ED with a 2- to 3-day history of abdominal pain, weakness, and nausea.}

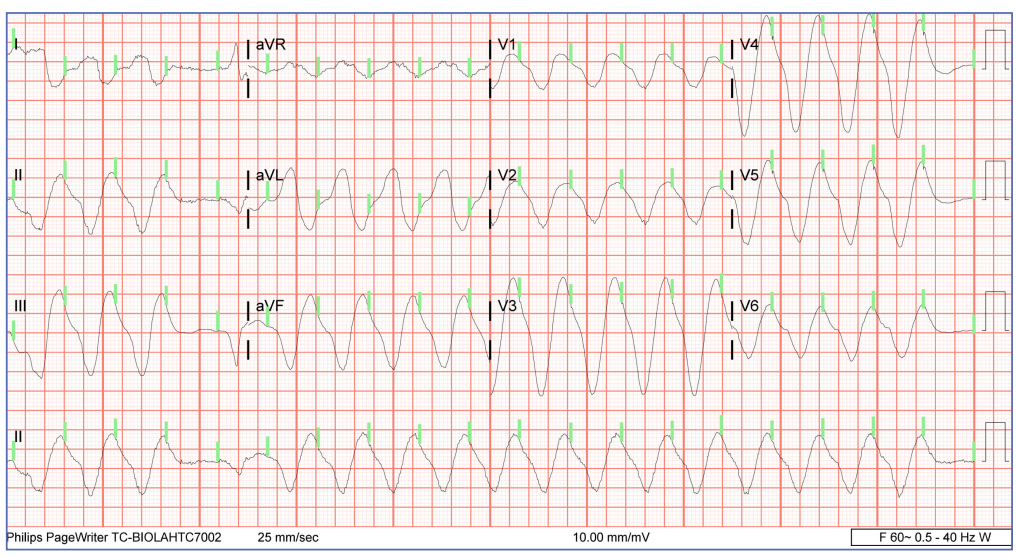

Figure 1. Initial electrocardiogram demonstrating wide complex tachycardia with pacer spikes.

Case

An 86-year-old woman, who recently had been seen in the same facility after a ground level fall, presented to the ED with to a 2- to 3-day history of vague abdominal pain, increasing weakness, nausea, and dry heaves.

Upon examination, the patient was unable to stand due to generalized weakness She arrived at the ED via emergency medical services. Her vital signs at presentation were significant for a systolic blood pressure (BP) of $90 \mathrm{~mm} \mathrm{Hg}$ with a wide complex tachycardia concerning for ventricu- lar tachycardia. The patient's other vital signers were: heart rate, 136 beats/min; respiratory rate 20 breaths/min; and pulse oximetry was $94 \%$ on 4 liters/min of oxygen via nasal cannula.

The patient's medical history was significant for atrial fibrillation and an indwelling pacemaker, for which she was chronically on flecainide and anticoagulation therapy with apixaban.

The initial electrocardiogram (ECG) revealed a wide complex rhythm with pacemaker spikes (Figure 1). Based on these findings, electrodes were placed on the patient in the event she required cardioversion. The patient was started on an amiodarone intravenous (IV) drip for presumptive ventricular tachycardia.

During the patient's evaluation in the ED, she experienced transient drops in BP, which were responsive to an IV fluid bolus of normal saline, and the amiodarone drip was discontinued. The patient's ECG findings were compared to previous ECG studies, as was her current medication list and prior health issues. After ruling-out other causes, flecainide toxicity was considered high in the differential, and she was given 1 ampule of bicarbonate IV, after which a second ECG showed heart rhythm con-

Dr Apfelbaum is a medical director, emergency medical services, Parker Adventist Hospital, Parker, Colorado. Ms Gerczynski is an undergraduate, pre-med student, Gustavus Adolphus College, Minneapolis, Minnesota. Dr Robertson is chairman, emergency and rescue department, Weber State University, Ogden, Utah. Mr Richey is executive director, Kolibri Forensics, Indianapolis, Indiana.

Authors' Disclosure Statement: The authors report no actual or potential conflict of interest in relation to this article.

DOI: 10.12788/emed.2018.0096 
verted from a wide-complex tachycardia to a paced rhythm, markedly improved from the initial ECG (Figure 2). Similarly, there was a marked improvement in BP.

An interrogation of the patient's pacemaker revealed an atrial flutter with a rate below detection for mode switch, with one-to-one tracking/pacing. The pacemaker was reprogrammed to divide the DDIR mode with detection rate at $120 \mathrm{~mm} \mathrm{Hg}$ with mode switch activated. This was felt to be consistent with flecainide toxicity precipitating the cardiac conduction issues.

Laboratory studies showed an elevated flecainide level at $1.39 \mathrm{mcg} / \mathrm{mL}$ (upper limits of normal of $1 \mathrm{mcg} / \mathrm{mL}$ ). Other studies showed worsening congestive heart failure, with a brain natriuretic peptide of $8,057 \mathrm{pg} / \mathrm{mL}$ and mild dehydration, with serum creatinine increased from her baseline of 0.9 to $1.38 \mathrm{mg} / \mathrm{dL}$.

The patient's abdominal pain was further evaluated and she was found to have acute cholecystitis. She was admitted to the intensive care unit with cardiology and general surgery consulting.

\section{Discussion}

Flecainide acetate was approved by the Food and Drug Administration in 1984..$^{1}$ It is a Vaughan-Williams class IC antiarrhythmic with a sodium channel blocker action used to treat supra ventricular arrhythmias. The CAST trial in 1989 investigated the efficacy of this class of antiarrhythmics, which resulted in a revision of its role. ${ }^{2}$ Based on this study, flecainide is not recommended for patients with structural heart disease or coronary artery disease. ${ }^{2,3}$ However, it is recommended as a first-line therapy for pharmacologic cardioversion and maintenance of normal sinus rhythm in patients with atrial fibrillation and supraventricular tachycardia ${ }^{4,5}$ without the above caveats.

Class IC agents produce a selective block at the sodium $(\mathrm{Na}+)$ channels, resulting in the slowing of cardiac conduction..$^{6,7}$ This high affinity for $\mathrm{Na}+$ channels combined

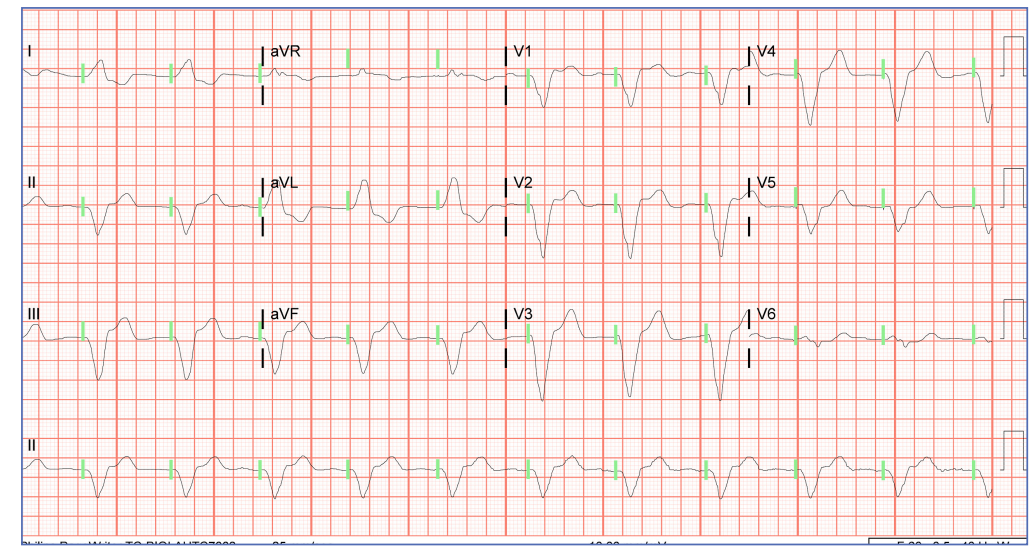

Figure 2. Second electrocardiogram demonstrating improvement of initial rhythm and pacer capture.

with slow unbinding kinetics during diastole explain the slowing of recovery time and prolongation of the refractory period. ${ }^{6,8,9}$ These electrophysiologic properties all can increase the PR, QRS, and QT interval duration. The QT interval is not significantly affected, as most of the QT prolongation is due to the QRS widening. ${ }^{6,10,11}$ Widening of the QRS by greater than $25 \%$ as compared to the baseline value is used as the threshold to decrease dosing or discontinue the use of flecainide. ${ }^{3}$

The toxic effects of flecainide on cardiac conduction can produce prolonged QRS duration of up to $50 \%$, and PR interval up to $30 \%$, especially in rapid heart rates. Signs of intoxication are difficult to discern owing to its nonspecific presentation. A well-documented, but under-recognized, presentation of flecainide toxicity is the transformation of atrial fibrillation to atrial flutter. ${ }^{5,7,9,11-13}$ The reported rate of this pro arrhythmic effect can be as high as $3.5 \%$ to $5 \% .^{14,15}$

Flecainide toxicity can occur secondary to chronic ingestion and may be precipitated in mild renal failure. The majority of flecainide is renally excreted and the halflife is 20 hours. Maximum therapeutic effect is seen between levels of 0.2 to $1 \mathrm{mcg} /$ $\mathrm{mL}$ with levels greater than 0.7 to $1 \mathrm{mcg} /$ $\mathrm{mL}$ associated with adverse effects. ${ }^{9}$ Systemic effects include dizziness and visual 
disturbances. A high degree of suspicion for flecainide toxicity is required when the patient's initial presentation is nonspecific. In this circumstance, real-time bedside interrogation of the pacemaker is invaluable. Early diagnosis and treatment minimizes the risk for adverse sequelae, including death. Treatment includes increasing the excretion of flecainide, symptomatic support (including pacemaker placement, intravenous fat emulsion, or extracorporeal circulatory support) and administration of sodium bicarbonate, to transiently reverse the effect of the sodium channel blockade, in severe cases. ${ }^{15-17}$

\section{References}

1. Hudak JM, Banitt EH, Schmid JR. Discovery and development of flecainide. Am J Cardiol. 1984;53(5):17B-20B.

2. Cardiac Arrhythmia Suppression Trial (CAST) Investigators. Preliminary report: effect of encainide and flecainide on mortality in a randomized trial of arrhythmia suppression after myocardial infarction. The Cardiac Arrhythmia Suppression Trial (CAST). N Engl J Med. 1989;321(6):406-412. doi:10.1056/ NEJM198908103210629.

3. Andrikopoulos GK, Pastromas S, Tzeis S. Flecainide: Current status and perspectives in arrhythmia management. World J Cardiol. 2015;7(2):76-85. doi:10.4330/wjc.v7.i2.76.

4. Camm AJ, Lip GY, De Caterina R, et al; ESC Committee for Practice Guidelines (CPG). 2012 focused update of the ESC Guidelines for the management of atrial fibrillation: an update of the 2010 ESC Guidelines for the management of atrial fibrillation. Developed with the special contribution of the European Heart Rhythm Association. Eur Heart J.
2012;33(21):2719-2747. doi:10.1093/eurheartj/ehs253.

5. Courand PY, Sibellas F, Ranc S, Mullier A, Kirkorian G, Bonnefoy E. Arrhythmogenic effect of flecainide toxicity. Cardiol J. 2013;20:203-205. doi:10.5603/ CJ.2013.0035

6. Holmes B, Heel RC. Flecainide. A preliminary review of its pharmacodynamic properties and therapeutic efficacy. Drugs. 1985;29(1):1-33.

7. Taylor R, Gandhi MM, Lloyd G. Tachycardia due to atrial flutter with rapid 1:1 conduction following treatment of atrial fibrillation with flecainide. $\mathrm{Br} \mathrm{Med}$ J. 2010;340:b4684.

8. Roden DM, Woosley RL. Drug therapy. Flecainide. N Engl J Med. 1986;315(1):36-41.

9. Levis JT. ECG diagnosis: flecainide toxicity. Perm J. 2012;16(4):53.

10. Hellestrand KJ, Bexton RS, Nathan AW, Spurrell RA, Camm AJ. Acute electrophysiological effects of flecainide acetate on cardiac conduction and refractoriness in man. Br Heart J. 1982;48(2):140-148.

11. Rognoni A, Bertolazzi M, Peron M, et al. Electrocardiographic changes in a rare case of flecainide poisoning: a case report. Cases J. 2009;2:9137. doi:10.1186/1757-1626-2-9137.

12. Nabar A, Rodriguez LM, Timmermans C, Smeets JL, Wellens HJ. Radiofrequency ablation of "class IC atrial flutter" in patients with resistant atrial fibrillation. Am J Cardiol. 1999;83(5):785-787, A10.

13. Kola S, Mahata I, Kocheril AG. A case of flecainide toxicity. EP Lab Digest. 2015;15(5).

14. Falk RH. Proarrhythmia in patients treated for atrial fibrillation or flutter. Ann Intern Med. 1992;117(2):141-150

15. Lloyd T, Zimmerman J, Griffin GD. Irreversible third-degree heart block and pacemaker implant in a case of flecainide toxicity. Am J Emerg Med. 2013;31(9):1418.e1-e2. doi:10.1016/j. ajem.2013.04.025.

16. Corkeron MA, van Heerden PV, Newman SM, Dusci L. Extracorporeal circulatory support in nearfatal flecainide overdose. Anaesth Intensive Care. 1999;27(4):405-408

17. Ellsworth H, Stellpflug SJ, Cole JB, Dolan JA, Harris CR. A life-threatening flecainide overdose treated with intravenous fat emulsion. Pacing Clin Electrophysiol. 2013;36(3):e87-e89. doi:10.1111/j.15408159.2012.03485.x. 